DO-TH-93/19EA

February 1994

\title{
Quantum Fluctuations of the electroweak sphaleron: Erratum and Addendum
}

\author{
J. Baacke H and S. Junker \\ Institut für Physik, Universität Dortmund \\ D-44221 Dortmund 50 \\ Germany
}

\footnotetext{
${ }^{1}$ e-mail: baacke@het.physik. uni-dortmund.de
} 
We have presented recently [1] an exact computation of the fluctuation determinant of the electroweak sphaleron. The results disagreed substantially from those of an earlier evaluation of this quantity [2]. Moreover none of the two exact computations agreed with analytical estimates [3, 4] that are expected to be good at small Higgs masses, essentially approximations in which higher gradient terms are neglected, a fact that has been repeatedly criticized (see e.g. [5]).

While we thought that possibly the gradient type expansions were to blame for this discrepancy, the failure of compatibility between approximations and exact results can be traced back to our treatment of the tadpole contributions. Indeed we removed all tadpole contributions to the Higgs field completely, in a misinterpretation of the renormalization and rescaling prescription of Refs. [2, 4]; however, a finite piece has to be restituted. In order to understand this point which has considerable numerical consequences we review shortly these contributions (see [6], especially Appendix C). This cannot be done consistently within the three dimensional asymptotic theory since the approximation of dismissing all but the lowest Matsubara frequency is not justified for a these divergent contributions.

The tadpole contribution including all Matsubara frequencies reads

$$
\mathcal{V}[\Phi]_{T a d}=\frac{T}{2} \sum_{i} \int \frac{d^{3} p}{(2 \pi)^{3}} \sum_{n=-\infty}^{\infty} \frac{1}{p^{2}+m_{i}^{2}+(2 \pi n T)^{2}} c_{i} \int d^{3} x\left(H_{0}^{2}(\vec{x})-1\right)
$$

Here $m_{i}$ are the masses circulating in the loop, $c_{i}$ are their couplings to the Higgs field and $H_{0}(\vec{x})$ is the Higgs profile. The coefficients $c_{i}$ can be identified as coefficients of the terms proportional to $H_{0}^{2}-1$ in the diagonal elements of the potential given in Appendix A of [1], they are given below. The momentum integral including the factor $T / 2$ can be rewritten [6]

$$
\int \frac{d^{3} p}{(2 \pi)^{3}}\left(\frac{1}{4 \sqrt{p^{2}+m^{2}}}+\frac{1}{2 \sqrt{p^{2}+m^{2}}} \frac{1}{\exp \sqrt{p^{2}+m^{2}} / T-1}\right)
$$

The first term is the $T=0$ contribution which goes into the Higgs mass renormalization. The second term can be expanded at high temperature as

$$
\frac{T}{\pi} \int x^{2} d x \frac{1}{\sqrt{x^{2}+m^{2} / T^{2}}} \frac{1}{\exp \sqrt{x^{2}+m^{2} / T^{2}}-1} \approx \frac{T^{2}}{24}-\frac{m T}{8 \pi}
$$


up to terms of order $\ln T$ or lower. The term quadratic in $T$ can be absorbed [2, 4] into the $T$ dependence of the vacuum expectation value of the Higgs field. The linear term is part of the well known $T \Phi^{3}$ term of the effective potential and without this contribution the latter is incomplete (see e.g. the discussion of this term in Appendix A of [5]). This is the reason why our data must fail to approach the estimate based on the effective potential.

Specifying the contributions of the different fields by [1] $m_{i}=M_{W}, c_{i}=$ $M_{W}^{2}$ for altogether six components of gauge boson and subtracted ghost fields, $m_{i}=M_{W}, c_{i}=\left(M_{H}^{2}+2 M_{W}^{2}\right) / 2$ for the three Goldstone boson fields and by $m_{i}=M_{H}, c_{i}=3 M_{H}^{2} / 2$ for the physical Higgs field we find a contribution to the effective action proportional to $T^{2}$

$$
\frac{T^{2}}{8}\left(3 M_{W}^{2}+M_{H}^{2}\right) \int d^{3} x\left(H_{0}^{2}-1\right)
$$

in agreement with standard results. The term linear in $T$ yields now

$$
-\frac{T}{8 \pi}\left(M_{W}\left(9 M_{W}^{2}+\frac{3}{2} M_{H}^{2}\right)+\frac{3}{2} M_{H}^{3}\right) \int d^{3} x\left(H_{0}^{2}-1\right) .
$$

This term is positive. Since the fluctuation determinant is related to $-\mathcal{V}_{1-\text { loop }} / T$ and the term is to be restituted in order to parallel the treatment of the effective potential, we find a large negative contribution to $\ln \kappa$.

We present in Table 1 our previous results with the completely removed tadpoles as $\ln \tilde{\kappa}$, in units of $(g v)^{6}$ as used in [2] and the results with the restituted piece as $\ln \kappa$. It is the latter one that is correct and that has to be compared with the $\Phi^{3}$ estimate derived from the effective potential which, if only the gauge loops are taken into account, takes the form [3]

$$
\frac{3 M_{W}^{3}}{4 \pi} \int d^{3} x\left(H_{0}^{3}-1\right)
$$

We give the corresponding estimates in the last row of Table 1, labelled " $\Phi^{3}$ ". This estimate differs from the one given in [2] by a factor $\sqrt{8}$ which is due to a mistake there: the term is originally [3] given for a Higgs field normalized to the vacuum expectation value $v$, while the one used in [2] has vacuum expectation value $v / \sqrt{2}$ ?. We observe that our corrected data and this estimate are now well consistent. Actually, since $\kappa$ is a dimensionful

\footnotetext{
${ }^{2}$ This statement is made with the agreement of Larry McLerran
} 
quantity $\ln \kappa$ depends on the scale used, therefore one can only expect that the exact data and the estimate become it parallel as $M_{H} \rightarrow 0$; the absolute agreement in this limit is somewhat fortitious.

Our corrected data, the analytic estimate and the data of Carson et al. [2] are presented in Figure 1, showing now a reasonable general agreement. Whether the remaining differences are within or outside the error margins of the involved numerical computations is a question that seems hard to answer. Unfortunately also the disagreement with the estimate of Ref. [四] persists.

\section{Acknowledgments}

One of us (J.B.) thanks K. Goeke and A. Ringwald for making him aware, at different times, of Ref. [5], W. Buchmueller and L. McLerran for most useful discussions, and the DESY for hospitality.

\section{References}

[1] J. Baacke and S. Junker, Phys. Rev. D49,2055 (1994)

[2] L. Carson,X. Li,L. McLerran and R.-T. Wang, Phys. Rev. D42, 2127 (1990)

[3] M. Shaposhnikov, Nucl. Phys. B287, 757 (1987)

[4] L. Carson and L. McLerran, Phys. Rev. D41, 647 (1990)

[5] M. Hellmund, J. Kripfganz and M. G. Schmidt, Heidelberg preprint HD-THEP-93-23, and private communications by many others

[6] Dolan and R. Jackiw, Phys. Rev. D9, 3320 (1974) 


\section{Table 1}

\begin{tabular}{|c||c|c|c|c|c|c|c|}
\hline$\xi$ & 0.4 & 0.5 & 0.6 & 0.8 & 1.0 & 1.5 & 2.0 \\
\hline$\lambda / g^{2}$ & .02 & .031 & .045 & .08 & .125 & .281 & .5 \\
\hline $\ln \widetilde{\kappa}$ & 2.02 & 1.73 & 1.52 & 1.34 & 1.32 & 1.46 & 1.55 \\
\hline $\ln \kappa$ & -46.80 & -31.00 & -22.30 & -13.64 & -9.64 & -5.96 & -5.14 \\
\hline$\Phi^{3}$ & -45.23 & -29.43 & -20.68 & -11.87 & -7.73 & -3.57 & -2.08 \\
\hline
\end{tabular}

\section{Figure Captions}

Fig. 1 The Fluctuation Determinant

We plot the logarithm of the fluctuation determinant $\kappa$ as a function of the ratio $\lambda / g^{2}$. Our corrected results are given as triangles, those of Ref. [2] as squares. The solid line is the estimate based on the effective potential. 
This figure "fig1-1.png" is available in "png" format from: http://arxiv.org/ps/hep-th/9402078v1 